\title{
Imaging Cell Death
}

\author{
André A. Neves ${ }^{1}$ and Kevin M. Brindle ${ }^{1,2}$ \\ ${ }^{I}$ Cancer Research United Kingdom Cambridge Institute, Li-Ka Shing Centre, Cambridge, United Kingdom; and ${ }^{2}$ Department of \\ Biochemistry, University of Cambridge, Cambridge, United Kingdom
}

There is currently a need for imaging methods capable of detecting cell death in tissues and the early onset of tumor cell death resulting from therapy. However, to date, no probe has been approved for routine imaging of cell death in the clinic. The challenge is to identify hallmarks of cell death, which have clinical relevance, and then to develop and validate imaging biomarkers for these hallmarks. We focus here on cell death imaging probes, which either have been trialed in the clinic or have significant promise, based on preclinical studies.

Key Words: apoptosis; necrosis; synaptotagmin; annexin; phosphatidylserine; PET; tumors; cardiac

J Nucl Med 2014; 55:1-4

DOI: 10.2967/jnumed.112.114264

"For death begins with life's first breath, and life begins at touch of death."

—William A. Dunkerley

Cell death plays an important role in tissue homeostasis and is a feature of disease, including cardiac disease and cancer. In acute myocardial infarction or cerebral stroke, the degree of apoptosis and necrosis can be significant and assessing both mechanisms of cell death can provide valuable diagnostic information on the extent of reversible tissue damage. In the case of cancer imaging, early tumor responses to therapy should enable more effective patient management, allowing rapid selection of the most effective treatment, minimizing deleterious side effects from ineffective treatments and reducing health care costs. The molecular alterations that occur in dying cells and that have been exploited as targets for imaging biomarkers are shown in Figure 1. These changes, which occur predominantly during apoptosis, include the externalization of phosphatidylserine to the outer leaflet of the plasma membrane bilayer; the activation of effector caspases, such as caspases- 3 and -7 ; the depolarization

Received Oct. 1, 2013; revision accepted Nov. 26, 2013.

For correspondence or reprints contact: André A. Neves, Cancer Research United Kingdom Cambridge Institute, Li-Ka Shing Centre, Robinson Way, University of Cambridge, Cambridge CB2 ORE, U.K.

E-mail: andre.neves@cruk.cam.ac.uk

COPYRIGHT @ 2014 by the Society of Nuclear Medicine and Molecular Imaging, Inc. of the plasma and mitochondrial membranes; and the loss of plasma membrane integrity, which occurs in necrosis.

\section{CELL DEATH PATHWAYS}

The physiology and pathways of cell death have been studied extensively (1). Apoptosis plays an important role in cell growth and differentiation, organismal development, and tissue homeostasis, particularly in the immune system. However, deregulation of cell death is associated with various diseases, including cancer, chronic cardiovascular and neurodegenerative diseases, and in autoimmunity. Historically, cell death has been classified, on the basis of morphologic criteria, as regulated (apoptosis) or nonregulated (necrosis). The current guidelines from the Nomenclature Committee on Cell Death recommend a classification based on biochemical and molecular events (2). Apoptosis, which is noninflammatory, is executed in response to internal (mitochondrial disruption) or external (death-domain receptor activation) stimuli. Necrosis is normally pathologic and inflammatory and occurs frequently as a response to overwhelming stress, including nonphysiologic $\mathrm{pH}$, extreme temperature, or osmotic pressure. Apoptosis and necrosis are not mutually exclusive but at two ends of a spectrum of cell death pathways, which are frequently highly regulated and interconnected (2). Late-stage apoptotic cells, for example, if not removed by immune cells, frequently undergo secondary necrosis (1). Additional forms of regulated cell death have been identified, including autophagy, necroptosis, mitotic catastrophe, and lysosomal-mediated programmed cell death (3), and there is plasticity and crosstalk between these cell death pathways in response to stress stimuli (2). Apoptosis and necrosis have important roles in cancer development, in the extent of myocardial damage after cardiac infarction and also in the way malignant tumors respond to chemo-, radio-, or hormonal therapy (3).

\section{IMAGING CELLULAR AND METABOLIC ALTERATIONS}

Cell death imaging agents fall into five main categories, according to the feature detected by the molecular imaging method (Fig. 1).

\section{Exposure of Phosphatidylserine}

Loss of the asymmetric distribution of the negatively charged amino-phospholipid phosphatidylserine is an early hallmark of apoptosis. Phosphatidylserine is the major anionic 


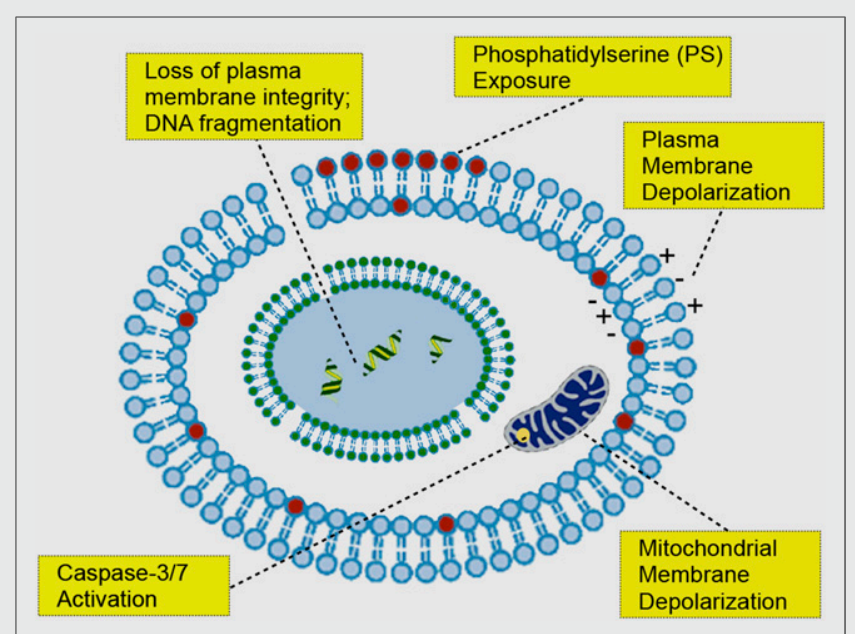

FIGURE 1. Illustration of main cellular events that historically have been exploited for imaging cell death.

phospholipid, representing $2 \%-10 \%$ of total cell phospholipid (1). Phosphatidylserine exposure occurs within a few hours of an apoptotic stimulus, by an active transport mechanism that leads to presentation on the cell surface of high concentrations of phosphatidylserine (50-200 million molecules of phosphatidylserine per apoptotic cell, which is equivalent to a cellular concentration of $20-100 \mu \mathrm{M})(4)$. This makes phosphatidylserine an abundant and accessible target for molecular imaging. Phosphatidylserine externalization is a hallmark of stressed and dying cells and a key signal for the removal of apoptotic cells by neighboring phagocytic cells, such as macrophages and neutrophils. Physiologic levels of phosphatidylserine on healthy cells are kept below the threshold detected by these phagocytic cells. Alternative cell death pathways, which show considerable crosstalk with apoptosis, including necroptosis and autophagy (2), also frequently involve phosphatidylserine exposure, making possible the imaging of multiple cell death pathways using this cell surface marker. Moreover, the final stages of most cell death pathways involve the loss of plasma membrane integrity, exposing phosphatidylserine on the inner leaflet of the plasma membrane bilayer to low-molecularweight phosphatidylserine-targeted imaging agents that can penetrate the necrotic cell plasma membrane.

Annexin- $\mathrm{V}(\mathrm{AnxV})$ is the most widely used imaging probe for phosphatidylserine-based detection of apoptosis, particularly using radionuclide imaging. AnxV is also one of the few cell death imaging agents that has reached phase II/III clinical trials (5). AnxV is a $36-\mathrm{kDa}$, nonglycosylated membrane protein, which can be produced by recombinant DNA technology (as Annexin A5). AnxV has a low nanomolar phosphatidylserinebinding affinity in the presence of calcium and is nonimmunogenic. Despite being a monomer in solution, AnxV forms trimer-based lattices on the surface of apoptotic cells, inducing strong cooperative phosphatidylserine-binding and driving cellular internalization. However, despite the early promise of ${ }^{99 \mathrm{~m}} \mathrm{Tc}-\mathrm{AnxV}$ probes for detecting apoptosis in cardiovascular disorders $(6)$ and for monitoring treatment response in head and neck cancer ( 1 ), AnxV has shown suboptimal biodistribution profiles, characterized by slow clearance and nonspecific binding to abdominal tissues (7). A second generation of site-specific mutants of AnxV has been proposed ( 8 ), yet the same problems persist, namely suboptimal pharmacokinetics and low specificity (9). These AnxV probes generated high background signal in the liver, kidneys, and gut, limiting the application of these probes for abdominal imaging and potentially contributing to organ and whole-body radiation exposure when radionuclide imaging is used (9).

Synaptotagmin-I (SynI) is a synaptic vesicle-associated protein, which binds, in the presence of $\mathrm{Ca}^{2+}$ ions, to the negatively charged phospholipids phosphatidylserine and phosphatidylinositol. We have proposed various probes for imaging tumor cell death based on the C2A domain of SynI (4). Recombinant C2A (14 kDa) can be expressed in Escherichia coli, in high yields $(\sim 100 \mathrm{mg} / \mathrm{L})$ as a glutathione S-transferase-tagged, dimeric construct ([GST-C $\left.2 \mathrm{~A}]_{2}, 84 \mathrm{kDa}\right)$. The isolated C2A domain and the GST-tagged dimer bind phosphatidylserine with high affinity $\left(\mathrm{K}_{\mathrm{D}}=20-60\right.$ and $1-5$ $\mathrm{nM}$ for C2A and (GST-C2A) ${ }_{2}$, respectively). (GST-C2A) has been labeled by us with superparamagnetic iron oxide nanoparticles or gadolinium chelates for MR imaging and by others with ${ }^{99 \mathrm{~m} T c}$ for SPECT and more recently with ${ }^{18} \mathrm{~F}$ for PET (10) for imaging of tumor cell death in mouse tumor models in vivo. These probes were labeled using the primary $\varepsilon$-amino groups of lysines, which are mostly on the GST tag (21 residues). However, this method yielded heterogeneous probe mixtures and could potentially lead to loss of phosphatidylserine-binding activity if any of the 14 lysine residues on $\mathrm{C} 2 \mathrm{~A}$ were modified. For this reason, we produced a much smaller $(16 \mathrm{kDa})$ site-directed mutant of the C2A domain (C2Am, S78C), containing a single cysteine residue distant from the phosphatidylserine-binding site, for site-specific modification (4). Our recent studies in vitro have shown that a fluorescently labeled version of $\mathrm{C} 2 \mathrm{Am}$ had a 5-fold lower binding to viable cells than a similarly labeled AnxV counterpart and, as a consequence, had better specificity for detection of apoptosis and necrosis (4).

Various small-molecule and peptide-based probes have been proposed for phosphatidylserine imaging (9). These smaller probes have the potential advantages of favorable biodistribution and pharmacokinetics, leading to faster blood clearance, efficient phosphatidylserine targeting of remote tumor regions, and high tumor-to-background contrast. Small imaging agents, however, frequently have low affinity or specificity for phosphatidylserine. Nevertheless, phosphatidylserine-targeted peptides have been used for detecting therapy response in murine models of melanoma and lymphoma (11) with ${ }^{18}$ F-FDG PET. Duramycin, a 19-aminoacid peptide with antimicrobial properties, has nanomolar affinity for phosphatidylethanolamine, which, like phosphatidylserine, is also externalized during apoptosis and repre- 
sents $20 \%-40 \%$ of total cell phospholipid (1). A single-step kit for ${ }^{99 \mathrm{~m}} \mathrm{Tc}$ labeling of duramycin has been proposed recently for SPECT imaging of apoptosis (12).

\section{Effector Caspase Activation}

Caspases are cysteine-aspartate proteases that play a pivotal role in the regulation of apoptosis. The intrinsic and extrinsic pathways of apoptosis converge on a small number of executioner caspases; caspases-3, -7, and -9 and several caspase inhibitors have been proposed for imaging of the activated (cleaved) enzymes. The isatin-sulphonamides are a family of indole derivatives with subnanomolar inhibitor binding constants for caspases-3 and -7 (5). The caspase-3/-7-specific PET radiotracer ${ }^{18} \mathrm{~F}-\mathrm{ICMT}-11$ ( ${ }^{18} \mathrm{~F}-(S)-1-((1-(2-$ fluoroethyl)1H-[1,2,3]-triazol-4-yl)methyl)-5-(2(2,4-difluorophenoxymethyl)-pyrrolidine-1-sulfonyl)isatin) has shown promise for the early detection of drug-induced tumor apoptosis in xenograft models of lymphoma $(13,14)$, breast, and colon (14) cancer, in which drug treatment increased probe retention in the tumors by 1.5 - to 2 -fold. Nevertheless, the authors noted that the rapid evolution of caspase-3/-7 activation renders imaging of this transient biomarker of apoptosis particularly challenging $(5,14)$. In fact, each drug or tumor model used in the study showed an ideal temporal window for imaging, which occurred between 6 and $24 \mathrm{~h}$ after treatment (14). This requirement to choose the best time to image may limit the clinical application of these agents. Moreover, isatin-based agents, which as cell-penetrating agents have amphipathic character, tend to be cleared by the hepatobiliary route, which frequently leads to a suboptimal biodistribution profile, with high abdominal uptake in the gallbladder, liver, and small intestine (14). Notwithstanding these 2 important limitations, the first human study using ${ }^{18}$ F-ICMT-11 has been conducted recently in 8 volunteers, indicating a dosimetry profile in healthy humans similar to that of other ${ }^{18} \mathrm{~F}$-PET tracers used in the clinic (e.g., ${ }^{18}$ F-FDG) yet also confirming the same predominant hepatobiliary probe clearance as was seen in the murine models (15).

\section{Mitochondrial Membrane Depolarization}

The collapse of mitochondrial membrane potential, which is a $\mathrm{Ca}^{2+}$-linked process, is important in apoptosis and necrosis. This occurs when nonspecific mitochondrial permeability transition pores open in the inner membrane, enabling free diffusion of small molecules, including protons. Mitochondrial permeability transition can occur as a result of $\mathrm{Ca}^{2+}$ overload in cardiac ischemia, or as a result of oxidative stress, or after treatment with the cytotoxic agents used in cancer therapy.

Arylphosphonium salts are lipophilic cations that-because of the highly negative mitochondrial transmembrane potential generated by oxidative phosphorylation-permeate the plasma membrane and accumulate at the inner membrane of intact mitochondria (1). During apoptosis, the loss of this potential leads to a reduction of phosphonium ion uptake. A PET voltage sensor based on ${ }^{18} \mathrm{~F}$-fluorobenzyl triphenyl- phosphonium has been proposed for monitoring therapy response in orthotopic prostate tumor models, generating an approximately $50 \%$ decrease in tumor signal $48 \mathrm{~h}$ after treatment (16). The same tracer has been used recently to delineate ischemic heart regions in a rat model of transient coronary occlusion (17), generating stable signal up to $45 \mathrm{~min}$ after injection, good lesion delineation, and matching with corresponding histology. However, ${ }^{18} \mathrm{~F}$-fluorobenzyl triphenylphosphonium, and other probes that target loss of mitochondrial membrane potential, generate negative contrast because they are released from cells on mitochondrial membrane depolarization. Moreover, cellular efflux mechanisms, mediated by multidrug resistance transporters, can lead to reduced cellular uptake through rapid probe efflux (9).

\section{Plasma Membrane Depolarization}

Several imaging probes have been proposed for apoptosis imaging, for which a mechanism has not yet been fully elucidated. The most notable of these imaging agents is the PET tracer based on 2-(5-fluoropentyl)-2-methyl malonic acid, produced by Aposense Ltd. ( $\left.{ }^{18} \mathrm{~F}-\mathrm{ML}-10,206 \mathrm{Da}\right)$ (7). These small-molecule agents are thought to detect plasma membrane depolarization and acidification of the apoptotic cell (7). The first study conducted in humans using ${ }^{18} \mathrm{~F}-\mathrm{ML}-10$ (18) showed favorable safety, stability, dosimetry, and biodistribution profiles, with predominantly renal clearance and fast elimination (half-life, $\sim 1 \mathrm{~h}$ in blood), supporting further clinical development. A recent study assessed response to whole-brain radiation therapy in 10 patients with brain metastases (19). A significant correlation was found between early changes in ${ }^{18} \mathrm{~F}$-ML-10 PET scans, 9-10 $\mathrm{d}$ after treatment, and size changes detected by contrast-enhanced MR imaging measurements at 6-8 wk after treatment (19). Multicenter clinical trials are currently taking place to assess ${ }^{18} \mathrm{~F}-\mathrm{ML}-10$ in multiple tumor types (19).

\section{DNA Fragmentation and Loss of Plasma Membrane Integrity}

DNA-damaging cytotoxic drugs or radiotherapy, which are used frequently in the treatment of tumors, can induce DNA double-strand breaks. The DNA-damage response signaling protein, $\gamma \mathrm{H} 2 \mathrm{AX}$, which binds at DNA double-strand breaks, has been imaged in vivo, using fluorophore- or ${ }^{111} \mathrm{In}$ DOTA-labeled antibodies, which were tagged with a cellpenetrating peptide sequence, Tat (20). After X-ray irradiation, or bleomycin treatment, the anti- $\gamma \mathrm{H} 2 \mathrm{AX}$-Tat probes produced signal enhancements in fluorescence and SPECT images of breast tumor xenografts, which were proportional to the treatment administered and provided a method for imaging DNA damage in vivo (20).

Plasma membrane destruction occurs late in various cell death pathways, including necrosis and necroptosis, leading to increased accessibility of imaging probes to intracellular macromolecules, such as $\mathrm{C} 2 \mathrm{Am}$ binding to phosphatidylserine. DNA fragmentation, which occurs late in apoptosis and in necrosis, has for a long time been imaged in vitro using planar intercalating agents (e.g., propidium iodide) and fluorescence 
microscopy. A gadolinium-labeled probe, based on TO-PRO-1 (quinolinium-4-[(3-methyl-2(3H)-benzothiazolylidene)methyl]1-[3-(trimethylammonio)propyl]-diiodide; Life Technologies), a positively charged intercalating agent, which interacts with phosphate groups in DNA, has been used for MR imaging of necrosis in a mouse model of myocardial infarction (21).

The possibility of imaging surrogate biomarkers of cell death pathways, using metabolic imaging, has provided a novel avenue for detecting response to tumor therapy. We have shown that the production of ${ }^{13} \mathrm{C}$-labeled malate from hyperpolarized $\left[1,4-{ }^{13} \mathrm{C}_{2}\right]$ fumarate can be used as a sensitive marker of tumor cell necrosis (22). In necrotic cells, in which the plasma membrane permeability barrier is compromised, fumarate enters the cell rapidly and is converted into malate in the hydration reaction catalyzed by the abundant enzyme fumarase. Using ${ }^{13} \mathrm{C}$ MR spectroscopic imaging, we showed in several different tumor models, treated with a variety of different drugs, that there was an increase in hyperpolarized $\left[1,4-{ }^{13} \mathrm{C}_{2}\right]$ malate production, compared with the untreated tumors (23). The technique, which was also shown to detect necrosis in normal tissues, such as the kidney (24), appears, under some circumstances, to be more sensitive than diffusion-weighted MR imaging at detecting low levels of diffuse necrosis (25).

\section{CONCLUSION}

Cell death detection remains one of the most important unsolved problems in clinical molecular imaging, and to date, no cell-death imaging agent has been approved for general clinical use. The main challenges include choice of a relevant molecular target, the accurate detection of the temporal and spatial occurrence of cell death, optimization of probe pharmacokinetics, and the minimization of nonspecific tissue binding and thus maximization of image contrast. Future translational research is likely to focus on diseases where assessment of the nature and extent of cell death plays an important role in personalized patient care, particularly in the detection of treatment-induced cell death in tumors and for imaging cell death in coronary atherosclerotic plaques.

\section{DISCLOSURE}

This work was supported by Cancer Research U.K. programme grant C197/A3514. No other potential conflict of interest relevant to this article was reported.

\section{REFERENCES}

1. Smith BA, Smith BD. Biomarkers and molecular probes for cell death imaging and targeted therapeutics. Bioconjug Chem. 2012;23:1989-2006.
2. Galluzzi L, Vitale I, Abrams JM, et al. Molecular definitions of cell death subroutines: recommendations of the Nomenclature Committee on Cell Death 2012. Cell Death Differ. 2012;19:107-120.

3. Kreuzaler P, Watson CJ. Killing a cancer: what are the alternatives? Nat Rev Cancer. 2012;12:411-424.

4. Alam IS, Neves AA, Witney TH, et al. Comparison of the C2A domain of synaptotagmin-I and annexin-V as probes for detecting cell death. Bioconjug Chem. 2010;21:884-891.

5. Nguyen QD, Challapalli A, Smith G, et al. Imaging apoptosis with positron emission tomography: 'bench to bedside' development of the caspase-3/7 specific radiotracer $\left[{ }^{18}\right.$ F]ICMT-11. Eur J Cancer. 2012;48:432-440.

6. Laufer EM, Winkens MHM, Narula J, et al. Molecular imaging of macrophage cell death for the assessment of plaque vulnerability. Arterioscler Thromb Vasc Biol. 2009;29:1031-1038.

7. Reshef A, Shirvan A, Akselrod-Ballin A, et al. Small-molecule biomarkers for clinical PET imaging of apoptosis. J Nucl Med. 2010;51:837-840.

8. Bauwens M, De Saint-Hubert M, Devos E, et al. Site-specific ${ }^{68} \mathrm{Ga}-$ labeled annexin A5 as a PET imaging agent for apoptosis. Nucl Med Biol. 2011;38:381-392.

9. Niu G, Chen X. Apoptosis imaging: beyond annexin V. J Nucl Med. 2010;51: 1659-1662.

10. Wang F, Fang W, Zhang MR, et al. Evaluation of chemotherapy response in VX2 rabbit lung cancer with ${ }^{18} \mathrm{~F}$-labeled C2A domain of synaptotagmin I. J Nucl Med. 2011;52:592-599.

11. Song S, Xiong C, Lu W, et al. Apoptosis imaging probe predicts early chemotherapy response in preclinical models: a comparative study with F-18-FDG PET. J Nucl Med. 2013;54:104-110.

12. Zhao M, Li Z. A single-step kit formulation for the ${ }^{99 \mathrm{~m}} \mathrm{Tc}$-labeling of HYNICDuramycin. Nucl Med Biol. 2012;39:1006-1011.

13. Nguyen QD, Smith G, Glaser M, et al. Positron emission tomography imaging of drug-induced tumor apoptosis with a caspase-3/7 specific $\left[{ }^{18} \mathrm{~F}\right]$-labeled isatin sulfonamide. Proc Natl Acad Sci USA. 2009;106:16375-16380.

14. Nguyen QD, Lavdas I, Gubbins J, et al. Temporal and spatial evolution of therapy-induced tumor apoptosis detected by caspase-3-selective molecular imaging. Clin Cancer Res. 2013;19:3914-3924.

15. Challapalli A, Kenny LM, Hallett WA, et al. ${ }^{18} \mathrm{~F}-\mathrm{ICMT}-11$, a caspase-3-specific PET tracer for apoptosis: biodistribution and radiation dosimetry. $\mathrm{J}$ Nucl Med. 2013;54:1551-1556.

16. Madar I, Huang Y, Ravert H, et al. Detection and quantification of the evolution dynamics of apoptosis using the PET voltage sensor F-18-fluorobenzyl triphenyl phosphonium. J Nucl Med. 2009;50:774-780.

17. Higuchi T, Fukushima K, Rischpler C, et al. Stable delineation of the ischemic area by the PET perfusion tracer F-18-fluorobenzyl triphenyl phosphonium after transient coronary occlusion. J Nucl Med. 2011;52:965-969.

18. Höglund J, Shirvan A, Antoni G, et al. F-18-ML-10, a PET tracer for apoptosis: first human study. J Nucl Med. 2011;52:720-725.

19. Allen AM, Ben-Ami M, Reshef A, et al. Assessment of response of brain metastases to radiotherapy by PET imaging of apoptosis with ${ }^{18} \mathrm{~F}-\mathrm{ML}-10$. Eur J Nucl Med Mol Imaging. 2012;39:1400-1408.

20. Cornelissen B, Kersemans V, Darbar S, et al. Imaging DNA damage in vivo using gammaH2AX-targeted immunoconjugates. Cancer Res. 2011;71:4539-4549.

21. Huang S, Chen HH, Yuan H, et al. Molecular MRI of acute necrosis with a novel DNA-binding gadolinium chelate: kinetics of cell death and clearance in infarcted myocardium. Circ Cardiovasc Imaging. 2011;4:729-737.

22. Gallagher FA, Kettunen MI, Hu DE, et al. Production of hyperpolarized $\left[1,4-{ }^{13} \mathrm{C}_{2}\right]$ malate from $\left[1,4-{ }^{13} \mathrm{C}_{2}\right]$ fumarate is a marker of cell necrosis and treatment response in tumors. Proc Natl Acad Sci USA. 2009;106:19801-19806.

23. Witney TH, Kettunen MI, Hu DE, et al. Detecting treatment response in a model of human breast adenocarcinoma using hyperpolarised $\left[1-{ }^{13} \mathrm{C}\right]$ pyruvate and [1,4- $\left.{ }^{13} \mathrm{C}_{2}\right]$ fumarate. Br J Cancer. 2010;103:1400-1406.

24. Clatworthy MR, Kettunen MI, Hu DE, et al. Magnetic resonance imaging with hyperpolarized $\left[1,4-{ }^{13} \mathrm{C}_{2}\right]$ fumarate allows detection of early renal acute tubular necrosis. Proc Natl Acad Sci USA. 2012;109:13374-13379.

25. Bohndiek SE, Kettunen MI, Hu DE, et al. Hyperpolarized ${ }^{13} \mathrm{C}$ spectroscopy detects early changes in tumor vasculature and metabolism after VEGF neutralization. Cancer Res. 2012;72:854-864. 